\title{
LXII. Some measurements of wave-lengths with a modified apparatus
}

\section{Lord Rayleigh O.M. Pres. R.S.}

To cite this article: Lord Rayleigh O.M. Pres. R.S. (1906) LXII. Some measurements of wavelengths with a modified apparatus, Philosophical Magazine Series 6, 11:65, 685-703, DOI: $10.1080 / 14786440609463487$

To link to this article: http://dx.doi.org/10.1080/14786440609463487

曲 Published online: 16 Apr 2009.

Submit your article to this journal \lceil

Џ Article views: 5

Q View related articles $\square$

4 Citing articles: 10 View citing articles 5 
Stefan's law for the black body could also not be considered conclusive. This state of affairs has been changed since we know, from the brilliant experiments of Lebedew and of Nichols and Hull, that the light-pressure does exist and does have, very approximately at all events, the value deduced a priori from Maxwell's theory. It still remains, however, highly desirable that another absolute radiation scale should be devised, so that we may have another and independent check on our high temperature measurements. Planck's formula rests on too many assumptions to be satisfactory theoretically; and Wien's displacement law, while its theoretical foundation is nearly as good as that of the StefanBoltzmann law, does not, by itself, offer a good method from the point of view of experimental accuracy.

U.S. Department of Agriculture,

Washington, Jan. 17, 1906.

LXII. Some Measurements of Wave-Lengths with a Modified Apparatus. By Lord RAYleigh, O.M., Pres. R.S.*

A $S$ the result of discussions held during the last three or A four years, it seems to be pretty generally agreed that the use of the diffraction-grating in fundamental work must be limited to interpolation between standard wavelengths determined by other means. Even under the advantageous conditions rendered possible by Rowland's invention of the concave grating, allowing collimators and object-glasses to be dispensed with, the accuracy attained in comparisons of considerably differing wave-lengths is found to fall short of what had been hoped. I think that this disappointment is partly the result of exaggerated expectations, against which in $1888 \dagger$ I gave what was intended to be a warning. Quite recently, Michelson $\ddagger$ has shown in detail how particular errors of ruling may interfere with results obtained by the method of coincidences ; but we must admit that the discrepancies found by Kayser $\S$ in experiments specially designed to test this question, are greater than would have been anticipated.

Under these circumstances, attention has naturally been directed to interference methods, and especially to that so

* Communicated by the Author.

$\dagger$ Wave Theory, Enc. Brit.; Scientific Papers, iii. p. 111, footnote.

\$ Astro-physical Journal, xviii. p. 278 (1903).

\Zeitschrift fiir wiss. Photographie, Bd. ii. p. 49 (1904). 
skillfully worked out by Fabry and Perot. In using an accepted phrase it may be well to say definitely that these methods have no more claim to the title than has the method which employs the grating. The difference between the grating and the parallel plates of Fabry and Perot is not that the latter depends more upon interference than the former, but that in virtue of simplicity the parallel plates allow of a more accurate construction. In Fabry and Perot's work the wave-lengths are directly compared with the green and red of cadmium; and they have obtained numbers, apparently of great accuracy, for artificial lights from vacuum-tubes containing various substances, $e . g$. mercury, for numerous lines from an iron arc, and also for various rays of the solar spectrum. While, so far as I can judge, there has been every disposition to receive with favour work which not only bears the marks of care but is explained with great discrimination, it must still be felt that, in accordance with an almost universal rule, confirmation by other hands is necessary to complete satisfaction. It was with this feeling that about a year ago I commeneed some observations of which I now present a preliminary account. I was not without hope that I might be able to introduce some variations which would turn out to be improvements, and which would, at any rate, promote the independence of my results.

In this method the interference rings utilized are of the kind first observed by Haidinger, dependent upon obliquity. Their theory is contained in the usual formule for the reflexion and transmission of parallel light by a "thin plate." Thus, if $\lambda$ be the wave-length of monochromatic light, $\kappa=2 \pi / \lambda, \delta$ the retardation, $e$ the reflecting power of the surface, we have, in the usual notation for the intensity of reflected light*,

and

$$
\mathrm{R}=\frac{4 e^{2} \sin ^{2}\left(\frac{1}{2} \kappa \delta\right)}{1-2 e^{2} \cos \kappa \delta+e^{4}}, \quad . \quad . \quad .
$$

$$
\delta=2 \mu t \cos \alpha^{\prime}, . \quad \text {. . . . . . }
$$

where $t$ denotes the thickness of the plate, $\mu$ the refractive index, and $\alpha^{\prime}$ the obliquity of the rays within the plate.

Another form of $(1)$ is

$$
\frac{1}{\mathrm{R}}=1+\frac{\left(1-e^{2}\right)^{2}}{4 e^{2} \sin ^{2}\left(l_{0} \kappa \delta\right)}, \quad . \quad . \quad . \quad . \quad \text {. }
$$

* See, for example, Wave Theory, Enc. Brit.; Scientific Papers, iii. pp. 64,65 . 
and from this we see that if $e=1$ absolutely;

$$
1 / R=R=1
$$

for all values of $\delta$. If $e=1$ very nearly, $\mathrm{R}=1$ nearly for all values of $\delta$ for which $\sin \left(\frac{1}{2} \kappa \delta\right)$ is not very small. In the light reflected from an extended source, the ground will be of the full brightness corresponding to the source, but it will be traversed by narrow dark lines. By transmitted light the ground, corresponding to general values of the obliquity, will be dark, but will be interrupted by narrow bright rings whose position is determined by $\sin \left(\frac{1}{2} \kappa \delta\right)=0$. In permitting for certain directions a complete transmission in spite of a high reflecting power $(e)$ of the surfaces, the plate acts the part of a resonator.

There is no transparent material for which, unless at high obliquity, $e$ approaches unity. In Fabry and Perot's apparatus the reflexions at nearly perpendicular incidence are enhanced by lightly silvering the surfaces. In this way the advantage of narrowing the bright rings is attained in great measure without too great a sacrifice of light. The plate in the optical sense is one of air, and is bounded by plates of glass whose inner silvered surfaces are accurately flat and parallel *. The outer surfaces need only ordinary flatness, and it is best that they be not quite parallel to the inner ones.

It will be seen that the optical parts are themselves of extreme simplicity; but they require accuracy of construction and adjustment, and the demand in these respects is the more severe the further the ideal is pursued of narrowing the rings by increase of reflecting power. Two forms of mounting are employed. In one instrument, called the interferometer, the distance between the surfaces-the thickness of the plateis adjustable over a wide range. In its complete developinent this instrument is elaborate and costly. The actual measurements of wave-lengths by Fabry and Perot were for the most part effected by another form of instrument called an étalon or interference-gauge. The thickness of the optical plate is here fixed; the glasses are held up to metal knobs, acting as distance-pieces, by adjustable springs, and the final adjustment to parallelism is effected by regulating the pressure exerted by these springs.

The theory of the comparison of wave-lengths by means of this apparatus is very simple, and it may be well to give it, following closely the statement of Fabry and Perot $\dagger$.

* The most important requirement is the equidistance of the surfaces, and would not be inconsistent with equal and opposite finite curvatures.

$\dagger$ Ann. de Chimie, xxv. p. 110 (1902). A good account is given in Baly's 'Spectroscopy.' 
Consider first the cadmium radiation $\lambda$. It gives a system of rings. Let $\mathrm{P}$ be the ordinal number of one of these rings, for example the first counting from the centre. This integer is supposed known. The order of interference at the centre will be $p=\mathrm{P}+\epsilon$. We have to determine this number $\epsilon$, lying ordinarily between 0 and 1 . The diameter of the ring under consideration increases with $\epsilon$; so that a measure of the diameter allows us to determine the latter. Lut $e^{*}$ be the thickness of the plate of air. 'The order of interference at the centre is $p=2 e / \lambda$. This corresponds to normal passage. At an obliquity $i$ the order of interference is $p \cos i$. Thus if $x$ be the angular diameter of the ring $\mathrm{P}, p \cos \frac{1}{2} x=\mathrm{P}$; or since $x$ is small,

$$
p=\mathrm{P}\left(1+\frac{x^{2}}{8}\right) \text {. }
$$

In like manner, from observations upon another radiation $\lambda^{\prime}$ to be compared with $\lambda$, we have

$$
p^{\prime}=\mathrm{P}^{\prime}\left(1+\frac{x^{\prime 2}}{8}\right)
$$

whence if $e$ be treated as an absolute constant,

$$
\frac{\lambda^{\prime}}{\lambda}=\frac{\mathrm{P}}{\mathrm{P}^{\prime}}\left(1+\frac{x^{2}}{8}-\frac{x^{\prime 2}}{8}\right) . \quad . \quad . \quad . \quad .
$$

The ratio $\lambda / \lambda^{\prime}$ is thus determined as a function of the angular diameters $x, x^{\prime}$ and of the integers $\mathrm{P}, \mathrm{P}^{\prime}$.

One of the principal variations in my procedure relates to the manner in which $\mathrm{P}$ is determined. MM. Fabry and Perot $\dagger$ say:-“L'étalon, une fois réglé, est mesuré en fonction des longueurs d'onde du cadmium, par les méthodes que nous avons précédement décrites ; l'emploi de interféromètre est nécessaire pour cela." I wished to dispense with the sliding interferometer, and there is no real difficulty in determining $\mathrm{P}$ without it. For this purpose we use a modified form of (4), viz. :-

$$
\frac{\mathrm{P}^{\prime}}{\mathrm{P}}=\frac{\lambda}{\lambda^{\prime}}\left(1+\frac{x^{2}}{8}-\frac{x^{\prime 2}}{8}\right), . . . .
$$

expressing $\mathrm{P}^{\prime} / \mathrm{P}$ as a function of $\lambda / \lambda^{\prime}$, regarded as known, and of the diameters. To test a proposed (integral) value of $P$, we calculate $\mathrm{P}^{\prime}$ from (5). If the result deviates from an integer by more than a small amount (depending upon the accuracy of the observations), the proposed value of $P$ is to be rejected.

* Now with an altered meaning.

$\dagger$ Loc. cit. p. 112. 
In this way, by a process of exclusion the true value is ultimately arrived at.

The details of the best course will depend somewhat upon circumstances. It will usually be convenient to take first a ratio of wave-lengths not differing much from unity. Thus in my actual operations the mechanical measure of the distance between the plates was $4.766 \mathrm{~mm}$., and the first optical observations calculated related to the two yellow lines of mercury. The ratio of wave-lengths, according to the measurements of Fabry and Perot, is 1.003650; giving after correction for the measured diameters 1.003641 as the ratio $\mathrm{P}^{\prime} / \mathrm{P}$. From the mechanical measure we find as a rough value of $P, P=16460$. Calculating from this, we get $\mathrm{P}^{\prime}=16519 \cdot 92$, not sufficiently close to an integer. Adding 22 to $\mathrm{P}$ we find as corresponding values

$$
\mathrm{P}=16482, \quad \mathrm{P}^{\prime}=16542 \cdot 00,
$$

giving $\mathrm{P}$ as closely as it can be found from these obserrations. This makes the value of $P$ for the cadmium-red ring observed at the same time about $14 £ 24$, and this should not be in error by more than \pm 30 .

Having obtained an approximate value of $P$ for the cadmium red, we may now conveniently form a table, of which the first column contains all the so far admissible (say 60) integral values of $P$. The other columns contain the results by calculation from (5) of comparisons between other radiations and the cadmium red. The second and third columns, for example, may relate to cadmium green and cadmium blue. These almost suffice to fix the value of $P$, but any lingering doubt will be removed by additional columns relating to mercury green and mercury yellow (more refrangible). An extract from the table (p. 690) may make the matter clearer.

Inasmuch as the ratio of cadmium red to cadmium green is $1 \cdot 2659650$, very nearly $5: 4$, only every fourth number for red is admissible on this ground alone. If we consider a number such as 14803 not excluded by the comparison with cadmium green, we see that while it would pass the mercury green test, it is condemned by the cadmium blue and still more by the mercury yellow test. The only possible value of $\mathrm{P}$ is found to be 14814 .

The criticism may probably suggest itself that, although other values of $\mathrm{P}$ may be excluded, the agreement of the row containing 14814 with integers is none too good. It is to be remembered that these observations were of a preliminary character, and were taken without the full precautions with 
regard to temperature afterwards found to be necessary. The formula at the basis of the calculation assumes that $e$, the thickness of the plate, is constant, but in fact it changes

\begin{tabular}{|c|c|c|c|c|}
\hline $\begin{array}{l}\mathrm{Cd} \\
\text { red. }\end{array}$ & $\begin{array}{c}\text { Cd } \\
\text { green. }\end{array}$ & $\begin{array}{c}\text { Cd } \\
\text { blue. }\end{array}$ & $\underset{\text { green. }}{\mathrm{Hg}}$ & $\underset{\text { yellow. }}{\mathrm{Hg}}$ \\
\hline $\begin{array}{r}14788 \\
9\end{array}$ & 18721.03 & $19836 \cdot 04$ & $17435 \cdot 24$ & \\
\hline $\begin{array}{r}14790 \\
1\end{array}$ & & $19840 \cdot 07$ & & \\
\hline & 1872609 & & & \\
\hline & 1872989 & $19844 \cdot 09$ & & \\
\hline 6 & $18731 \cdot 15$ & $19848 \cdot 19$ & & \\
\hline 8 & .................. & $190+012$ & & \\
\hline $\begin{array}{r}9 \\
14800\end{array}$ & $\begin{array}{r}18734 \cdot 95 \\
\ldots \ldots \ldots \ldots .\end{array}$ & $\begin{array}{l}19850 \cdot 80 \\
19852 \cdot 14\end{array}$ & & \\
\hline $\begin{array}{l}1 \\
2\end{array}$ & engen & & & \\
\hline $\begin{array}{l}3 \\
4 \\
5\end{array}$ & 18740.01 & $19856 \cdot 16$ & $17459 \cdot 04$ & $16518 \cdot 68$ \\
\hline 6 & & $19860 \cdot 19$ & & \\
\hline 8 & $\begin{array}{l}18745 \cdot 08 \\
\ldots \ldots \ldots \ldots \ldots\end{array}$ & 1986287 & & \\
\hline $\begin{array}{r}9 \\
14810\end{array}$ & $18748 \cdot 88$ & & & \\
\hline & $18750 \cdot 14$ & 19866.90 & $17462: 36$ & \\
\hline $\begin{array}{l}4 \\
5\end{array}$ & $18753 \cdot 94$ & $19870 \cdot 92$ & $17465 \cdot 90$ & 16530.96 \\
\hline
\end{tabular}

with temperature. On this account alone erroneous results will be obtained unless the observations are well alternated, so as to eliminate such effects. The numbers finally arrived at, in substitution for the row in the table, are

$$
\text { 14814, 18753•95, 19870.95, 17465·97, 16531·00. }
$$

The deviations from integers still outstanding have their origin in a complication which must be admitted to be a drawback to the method and might at first sight be estimated even more seriously. The optical thickness $e$ of the plate, on which everything depends, is not really constant, as bas been assumed, when we pass from one part of the spectrum to another somewhat distant from the first. The question is discussed by Fabry and Perot. If, to take account of this 
factor, we denote the thicknesses for the two wave-lengths by $e_{\lambda}, e_{\lambda^{\prime}}$, we have

$$
\frac{p^{\prime}}{p}=\frac{e_{\lambda}}{e_{\lambda}} \frac{\lambda}{\lambda^{\prime}}
$$

and accordingly in place of (5)

$$
\mathrm{P}^{\prime}=\frac{\lambda e_{\lambda^{\prime}}}{\lambda^{\prime} e_{\lambda}}\left(1+\frac{x^{2}}{8}-\frac{x^{\prime 2}}{8}\right) . . \quad . \quad . \quad .
$$

But although I was prepared to find the calculated values of $\mathrm{P}^{\prime}$ differing somewhat from integers, I was disturbed by the amount and at first by the direction of the difference. For in their paper of 1899 * Fabry and Perot remark:- "Le surface optique du métal pour la radiation rouge est, par suite, située un peu plns profondément dans le métal que celle de la lumière verte, et à une distance de $4 \mu \mu$." At this rate $e_{\lambda}$ (red) would exceed $e_{\lambda^{\prime}}$ (green), and the introduction of the new factor in (6) would increase, and not remove, the discrepancy. It would seem, however, that the passage above quoted is in error and inconsistent with the discussion given in the later payer $t$, itself indeed embarrassed by several misprints $\ddagger$.

The amount of the correction required to bring the number for cadmium green up to an integer-about $2 \frac{1}{2}$ parts in a million-is $2 \frac{1}{2}$ times as great as one would expect from Fabry and Perol's indications $\$$. As to this, it may be observed that the wave-lengths employed in the calculation of the cadmium radiations are those of Michelson, and were obtained by a method free of the complication now under discussion. If these are correct, as there is no reason to doubt, and if there is no mistake in the identification of the ring-and there can be none here-it follows that the change of optical thickness in passing from red to green is determined by the numbers given and may be used to correct ratios of wavelengths not previously known with precision.

If we wish to make the results of the present method entirely independent, we must obtain material from observation sufficient to allow the variation of thickness with wavelength to be eliminated, that is, we must use the same silvered

* Ann. de Chimie, xvi. p. 311.

+ L. c. pp. 120-124.

$\mp$ Of these it may be worth while to note that the sign of $6.6 \mu \mu$ on p. 123 , line 5 should apparently be - instead of + .

$\$$ It is known that the effect depends upon the thickness of the silver films; perhaps also upon the process used in silvering and upon the condition of the surfaces in other respects. Surfaces that have stood some time in air are almost certain to be contaminated with layers of volatile greasy matter. 
plates at two different distances. In Fabry and Perot's work the sliding interferometer was employed ; the silvered surfaces were brought to very small distances, and the coincidences of two band systems, e.g. cadmium red and cadmium green, were observed, the telescope being focussed upon the plate, and not as before for infinity. It appears that excellent results were obtained in this way, affording material for eliminating the complication due to change of optical thickness.

It is rather simpler, in principle, and has the incidental advantage of allowing the sliding interferometer to be dispensed with, if we follow the same method for the small as for the greater distance. If the calculation be conducted on the same lines as before by means of (5), we ought to obtain the same fractional part again in the value of $\mathrm{P}^{\prime}, e . g . \cdot 95$ for cadmium green referred to cadmium red. For, as we see from (6), the proportional error in $\mathrm{P}^{\prime} / \mathrm{P}$ as calculated from (5) is $\left(e_{\lambda^{\prime}}-e_{\lambda}\right) / e_{\lambda}$. In the second set of operations, writing $\boldsymbol{\eta}$ for $e$, we find as the proportional error $\left(\eta_{\lambda^{\prime}}-\eta_{\lambda}\right) / \eta_{\lambda}$, in which $\eta_{\lambda^{\prime}}-\eta_{\lambda}=e_{\lambda^{\prime}}-e_{\lambda}$; so that the proportional errors are as $\eta_{\lambda}: e_{\lambda}$, or inversely as $\mathrm{P}$ or $\mathrm{P}^{\prime}$. Thus the absolute error in $\mathrm{P}^{\prime}$, as calculated from (5), is unaffected by the change of $e$ to $\eta$. If the fractional part is not recovered, within the limits of error, it is a proof that the assumed ratio of wave-lengths calls for correction, and the discrepancy gives the means for effecting such correction.

The above procedure is the natural one, when it is a question of identifying a ring or of confirming ratios of wave-lengths already presumably determined with full accuracy; but when the object is to find more accurately wave-lengths only roughly known, it has an air of indirectness. Otherwise, we have as before,

$$
2 e_{\lambda}=p \lambda, \quad 2 e_{\lambda^{\prime}}=p^{\prime} \lambda^{\prime} ;
$$

and again for a smaller interval between the surfaces,

$$
2 \eta_{\lambda}=\pi \lambda, \quad 2 \eta_{\lambda}=\pi^{\prime} \lambda^{\prime} .
$$

Hence and

$$
\begin{array}{r}
2\left(e_{\lambda}-\eta_{\lambda}\right)=(p-\pi) \lambda, \quad 2\left(e_{\lambda^{\prime}}-\eta_{\lambda^{\prime}}\right)=\left(p^{\prime}-\pi^{\prime}\right) \lambda^{\prime} ; \\
e_{\lambda}-\eta_{\lambda}=e_{\lambda^{\prime}}-\eta_{\lambda^{\prime}}, \quad \text { so that } \\
\frac{\lambda}{\lambda^{\prime}}=\frac{p^{\prime}-\pi^{\prime}}{p-\pi} \ldots . \quad . \quad . \quad . \quad .
\end{array}
$$

Hence $p, \pi, p^{\prime}, \pi^{\prime}$ are the ordinal numbers at the centre. They are to be deduced, as before, from the integral numbers proper to the rings actually observed and from the measured angular diameters of these rings. 
It is obvious that $p$ and $\pi$ must not be nearly equal. If $p$ be the larger number corresponding to the greater interval, $\pi$ should not exceed $\frac{1}{4} p$. On the other hand, too great a reduction of $\pi$ would lead to difficulties on account of the increased angular diameter of the rings. Perhaps it was for this reason that Fabry and Perot adopted an altered course. In my experiments the longer interval was, as already mentioned, about $5 \mathrm{~mm}$., and the shorter interval was about $1 \mathrm{~mm}$., so that the angular diameter of the rings was rather more than doubled in the latter case.

The facility with which angular diameters larger than usual could be observed is due, in part at any rate, to the special construction of my apparatus. M.M. Fabry and Perot employed a fixed interference-gauge and a fixed telescope, measuring the diameters of the rings by an eyepiece micrometer. 'There are, I think, some advantages in a modified arrangement, whereby it becomes possible to refer the rings to a wire fixed in the optic axis of the telescope. To this end the wire is made vertical, and the rings are brought to coincidence with it by a rotation of the gauge, which is mounted upon a turntable giving movement round a vertical axis. The middle plane of the gange is vertical and adjusted so as to include the axis of rotation. In this way of working the reference wire is backed always by the same light, whether opposite sides of one ring or of different rings are under observation. It is perhaps a more important advantage that the same part of the object-glass is always in use, and to a better approximation the same parts of the plates of the gauge. The diaphragm which limits the latter should be as close to the plates as possible (or to their image near the eye), but when the multiple reflexions are taken into account it is impossible to secure that exactly the same part should always be in action.

The revolving turntable carried with it a thick strip of plate-glass upon which was scratched a radial line. The point observed described a circle of 10 inches radius, and the rotation was measured by means of a travelling microscope reading to .001 inch. The angles involved are sufficiently small to allow the diameter of a ring to be taken as proportional to the difference of readings at the microscope.

As regards the gauge itself, the plates are by Brashear. For the mounting of the $5 \mathrm{~mm}$. gauge, which is of brass, I am indebted to my son $\mathrm{Mr}$. R. J. Strutt. The $1 \mathrm{~mm}$. gauge is of iron and was made by my assistant Mr. Enock. They are much after the design of Fabry and Perot. For the final adjustment to parallelism the eye is moved in various directions 
across the line of vision so as to bring different parts of the plates into action, and for this purpose it may be desirable to increase the aperture. A dilatation of the rings means that the corresponding parts of the plates need approximation by additional pressure. The aperture employed in the actual measurements was of about $9 \mathrm{~mm}$. diameter.

The (achromatic) object-glass of the telescope is of 15 inches focus. In rigid connexion with it is the vertical reference wire accurately adjusted to focus, and close to the wire a small frame suitable for carrying the horizontal slits (cut out of thin sheet zinc) necessary for the isolation of the various colours*. The eyepiece is a single lens of 5 inches focus, mounted independently, so that it ean be re-adjusted without fear of disturbing the object-glass and reference wire. The change of position required for the best seeing in passing from red to blue or even from red to green is so great as to occasion surprise that good results can be attained in the absence of such a provision $\dagger$.

The separation of the colours was usually effected by directvision prisms held between the eyepiece and the eye. Of these two were available. The larger containing (in all) three prisms was usually the more convenient, but sometimes a smaller and more dispersive combination containing five prisms was preferred. It is better to use more dispersion than unduly to narrow the slit. The refracting edges of the prisms are, of course, horizontal. In order to secure that the proper parts of the ring systems should be visible, the axis of the telescope was adjusted in the vertical plane with substitution for the slit of a horizontal wire coincident with the middle line of the former.

The advantage of this arrangement is that the ring systems (or at least so much of them as is necessary) of the various radiations emitted by one source of light are all in view at the same time.

In some cases, direct-vision prisms held between the 5-inch eye-lens and the eye do not suffice. The soda lines, for example, require a high dispersion. Even the yellow lines of mercury, which are about three times as far apart as the soda lines, could not be fully separated by the prisms already spoken of. Here a good deal depends upon chance. If the rings of one mercury system happen to bisect approximately those of the other system, both can be measured in the interferometer-gauge, and the only question which remains open

* Fabry and Perot, C. R. March 27, 1904.

$\dagger$ Especially in using the method of coincidences. I ought perhaps to mention that my eyes have now very little power of accommodation. 
is the distinction of the two systems. For this purpose a prism of moderate power, by which one system is lifted a little relatively to the other, suffices. If, however, the two ring systems chance to be pearly in coincidence, a much more powerful dispersion is required in order to measure them separately.

In such cases recourse was had to a special direct-vision prism of glass and bisulphide of carbon through which a selected ray of the spectrum passes without refraction at all at any of the surfaces *. In this instrument the upper edge of the beam traverses 20 inches of glass and the lower edge 20 inches of bisulphide of carbon. This prism cannot be inserted between the eyepiece already described and the eye, which latter must be placed at the image of the object-glass. Additional lenses are therefore required. These are merely ordinary spectacle-lenses and constitute a telescope of unit magnifying power. A more precise description is postponed, as $\mathrm{I}$ am not sure that $\mathrm{I}$ have as yet hit upon the best arrangement. It may suffice to say that with this instrument rings formed of spectral rays even closer than the soda lines could be readily separated, and that without too great a contraction of the slit limiting the visible portion of the rings.

The source of light, sometimes very small, was focussed upon the diaphragm at the gauge, and it is necessary that the aperture be completely filled with light. This gives the ratio between the distances of the lens from the source $(u)$ and from the gauge $(v)$. Again, the angular diameter of the field of light, which must not be too small, fixes the ratio of the aperture of the lens to $v$; so that only the absolute scale of the three quantities is left open. It is desirable that the lens be achromatic. I have used a one-inch lens from a small opera-glass, and this worked well with $u=2 \frac{1}{2}$ inches and $v=4$ feet.

As sources of light in experiments involving bigh interference, vacuum-tubes are by far the most convenient, and their introduction is one of the many services which Optics owes to Prof. Michelson. At the head of the list stands the helium tube, both on account of its not requiring to be heated and also of the brilliancy of the yellow radiation. Hitherto, however, the wave-lengths have not been measured with the highest accuracy. The tube that I have employed was made some years ago by my son and had already seen a good deal of service in experiments designed to answer the question: "Is Rotatory Polarization influenced by the Earth's

* Nature, lx. p. 64 (1899); Scientific Papers, iv. p. 394. 
Motion?*" From the overpowering brilliancy of the yellow line, it may be inferred that the pressure is not very low. Mercury too, for which the principal wave-lengths have been determined with great accuracy by Fabry and Perot, is convenient as requiring only a very moderate heating; and cadmium, in spite of the higher temperature demanded, is indispensable. Not only is the cadmium red by general consent the ultimate standard, but a comparison of the red and green ring systems, even without a prism, gives rapid information as to the condition of the gauge, slightly variable from time to time on account of temperature and of necessary readjustments. Thus, in most of my observations, the red ring under measurement was in very approximate coincidence with a green ring. If, owing to rise of temperature, this ring had so far expanded as to make it advisable to substitute the next interior one, there could still be no uncertainty as to the order (one higher) of the ring actually under observation.

As cadmium tubes appear to have been found troublesome, it may be well to describe a simple construction specially adapted to private workers whose skill in glass-blowing is limited. It was thought that alloying and consequent expansion of platinum sealings was a likely source of difficulty, and these were accordingly dispensed with. The diagram exhibits half the complete tube. The working capillary A, the enlargement $\mathrm{B} \mathrm{D}$, and the lateral tube $\mathrm{C}$ for attachment

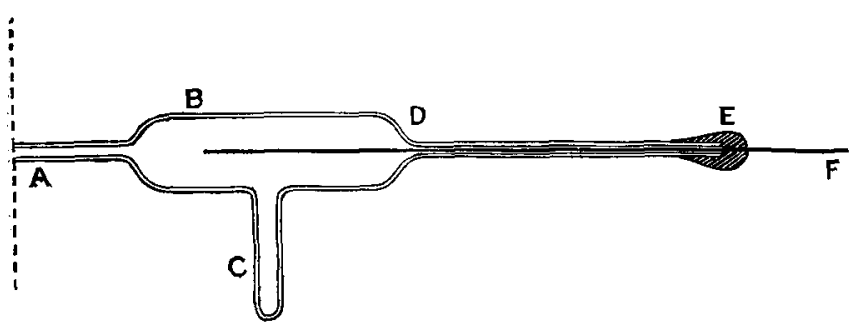

to the pump are much as usual. But the enlargement is continued by a second capillary DE, perhaps $1 \frac{1}{2} \mathrm{~mm}$. in diameter and $15 \mathrm{~cm}$. long, through which passes with approximate fit a straight aluminium wire, serving as electrode. The air-tight joint at $\mathbf{E}$ between the wire and the glass is made with sealing-wax. The length $\mathrm{DE}$ must be sufficient

* Phil. Mag. vol. iv. p. 215 (1902). 
to allow E to remain cool, although D, enclosed in a copper case, is hot enough to keep the cadmium vapour uncondensed. The lateral tube $\mathrm{C}$ projects from the case, and the cadmium condensed in it may need to be driven back from time to time by temporary application of the flame of a spirit-lamp or bunsen-burner.

This construction, used with cadmium, mercury, and thallium, has so far answered my expectations Cadmium tubes, apart from failures by cracking, are said often to deteriorate rapidly. My experience did not contradict this; for after four or five evenings' work the red radiation, which at first had been very brilliant, was no longer serviceable, although the green did not seem to have suffered much. At this stage the tube was re-exhausted and then appeared to behave differently, the red radiation being much better maintained. One must suppose that something deleterious had been emitted and been pumped away. There is much in the behaviour of vacuum-tubes which at present defies explanation.

To excite the electric discharge a large Ruhmkorff, actuated by five small storage-cells, was usually employed. Sometimes, especially in the comparison of the cadmium radiations, an alternate current was substituted; but there was no perceptible difference in the measurements. In this case a transformer of home construction was fed from a De Meritens magneto machine.

The radiations from zinc (and occasionally from cadmium) were obtained by an arrangement similar to Fabry and Perot's "trembler" *. The behaviour was very capricious. Sometimes, even when actuated by five secondary cells only, the zine rings were magnificent; but the deterioration was usually rapid as the zinc points lost their metallic surfaces. This change appears to be independent of oxidation. When the current was from a dynamo giving about 80 volts, the apparatus was less troublesome, but even then required careful management. The fineness of the points needs to be accommodated to the current employed.

As an example of the observations and calculations therefrom, I will take a series of Dec. 20,1905 , relative to the three radiations from the cadmium vacuum-tube. In this series the temperature conditions were more favourable than usual.

* C. R. 130. p. 406 (1900).

Phil. Mag. S. 6. Vol. 11. No. 65. May 1906. 
Cadmium (5 mm. gauge).

\begin{tabular}{|c|c||c|c||c|c|}
\hline \multicolumn{2}{|c|}{ RED. } & \multicolumn{2}{c||}{ GREEN. } & \multicolumn{2}{c|}{ BLUE. } \\
\hline Right. & Left. & Right. & Left. & Right. & Left. \\
$\cdot 398$ & $\cdot 221$ & $\cdot 401$ & $\cdot 217$ & $\cdot 406$ & $\cdot 213$ \\
.399 & $\cdot 221$ & $\cdot 402$ & $\cdot 217$ & $\cdot 404$ & $\cdot 211$ \\
$\cdot 399$ & $\cdot 220$ & $\cdot 402$ & $\cdot 216$ & $\cdot 406$ & $\cdot 210$ \\
\hline$\cdot 3987$ & $\cdot 2207$ & $\cdot 4017$ & $\cdot 2167$ & $\cdot 4053$ & $\cdot 2113$ \\
\hline \multicolumn{2}{|c|}{ Diff. $=\cdot 1780$} & & Diff. $=\cdot 1850$ & \multicolumn{2}{|c|}{ Diff. $=\cdot 1940$} \\
\hline
\end{tabular}

The numbers entered are the actual readings of the microscope in inches for settings on the right and left sides of the rings. Each horizontal row constitutes really a complete set. In order to eliminate temperature effects as far as possible, the readings are taken in a certain sequence. Thus in the first row the sequence was Red (R), Green (R), Blue (R), Blue (L), Green (L), Red (L). The differences, representing the diameters of the rings, are thus appropriate to the middle of the time occupied. If, as happened here and usually, the temperature was rising, so that the rings dilated, the first reading $(\cdot 398)$ on the red is too small, but the error is compensated in the last reading $(\cdot 221)$, which is equally too small. As a matter of convenience the next row would be taken in the reverse order, beginning with a repetition of Red (L), and so on.

Since the radius of the circle described by the point of observation is 10 inches, the angular diameters $(x)$ of the rings are as follows :-

\begin{tabular}{|c|c|c|c|}
\hline & RED. & Grein. & Blue. \\
\hline$x \ldots \ldots \ldots$ & .01780 & .01850 & .01940 \\
\hline$x^{2}$ & $10^{-4} \times 3 \cdot 168$ & $10^{-4} \times 3.422$ & $10^{-4} \times 3 \cdot 764$ \\
\hline$\frac{1}{8} x^{2}$ & $10^{-4} \times \cdot 3960$ & $10^{-4} \times \cdot 4277$ & $10^{-4} \times \cdot 4705$ \\
\hline Diff. .... & ............. & $10^{-4} \times 0317$ & $10^{-4} \times 0745$ \\
\hline
\end{tabular}

The calculation now proceeds by means of (5). If $P$ refer to cadmium red and $\mathrm{P}^{\prime}$ to green, we have with Michelson's 
values of the wave-lengths:

$$
\frac{\mathrm{P}^{\prime}}{\mathrm{P}}=1 \cdot 2659650(1-\cdot 00000317)=1 \cdot 2659610,
$$

which with $\mathrm{P}=14814$ gives

$$
\mathrm{P}^{\prime}=14814+3939 \cdot 945=18753 \cdot 945 \text {. }
$$

In like manner for the blue referred to the red,

$$
\frac{\mathrm{P}^{\prime}}{\mathrm{P}}=1 \cdot 3413733(1-\cdot 00000745)=1 \cdot 3413633,
$$

whence

$$
\mathrm{P}^{\prime}=14814+5056 \cdot 955=19870 \cdot 955 .
$$

The wave-lengths of the various radiations from a single source can thus be compared with great ease, and but little fear of temperature error. A set of observations from which this error is practically eliminated can be made in a short time and a few repetitions give all the security necessary. But the situation is not so favourable when we compare radiations from different sources. More time is occupied and there is corresponding opportunity for temperature change. It is necessary to alternate the observations, taking the first source twice and the second once, or preferably the first three times and the second twice. Even with this precaution I believe that temperature change was the principal source of error in the results of a single evening's work.

In the observations with an interval of one millimetre between the silvered surfaces, the influence of temperature is of course much less perceptible. For a similar reason the identification of the rings is a much easier matter. I will give as a specimen a series of operations (Feb, 9) in which helium was compared with cadmium. The first and third sets, each containing a repetition, related to cadmium ; the second set (twice repeated) related to helium. Only the mean diameter for each set is here recorded :-

Cadmium.

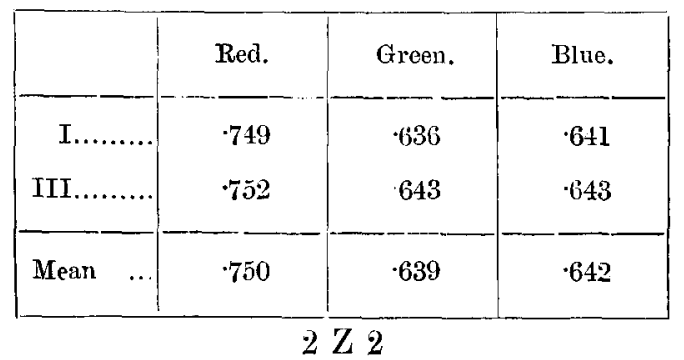


Lord Rayleigh: Some Measurements of

Helium.

\begin{tabular}{|c|c|c|c|c|c|c|c|}
\hline & $\begin{array}{c}\text { Red } \\
7065\end{array}$ & $\begin{array}{c}\text { Red } \\
6678\end{array}$ & $\begin{array}{c}\text { Yellow } \\
5876\end{array}$ & $\begin{array}{c}\text { Green } \\
5016\end{array}$ & $\begin{array}{c}\text { Green } \\
4922\end{array}$ & $\begin{array}{c}\text { Blue } \\
\mathbf{4 7 1 3}\end{array}$ & $\begin{array}{c}\text { Violet } \\
\mathbf{4 4 7 2}\end{array}$ \\
\hline II. ...... & 704 & 673 & 721 & 630 & 684 & 665 & 6637 \\
\hline
\end{tabular}

The first question is as to the ratio $\mathrm{P}^{\prime} / \mathrm{P}$ derived by (5) from these numbers for cadmium. The integral value of $P$ for the cadmium red ring was 3328. From this we find

$$
\begin{aligned}
& \mathrm{P}^{\prime} \text { cad. green }=4213 \cdot 946, \\
& \mathrm{P}^{\prime} \text { cad. blue }=4464 \cdot 935,
\end{aligned}
$$

on the basis of Michelson's wave-lengths. For the green the fractional part is practically identical with that deduced above from one set of observations with the $5 \mathrm{~mm}$. gauge. In the case of the blue the fractional part is now distinctly lower.

The above are the results of work on single evenings. On the mean of all the comparisons with the two intervals there resulted :-

Cadnium.

\begin{tabular}{|c|c|c|}
\hline & $5 \mathrm{~mm}$. & $1 \mathrm{~mm}$. \\
\hline Red ............. & 14814 & 3328 \\
Green ........... & 18753.95 & 4213.95 \\
Blue.............. & 19870.95 & 4464.94 \\
\hline
\end{tabular}

As already explained the agreement of the fractional parts constitutes a complete verification of Michelson's ratios of wave-lengths, accurate to one part in 2 millions in the case of red and blue and to a still closer accuracy in the case of red and green. And it appears further that the phasechanges, upon which depend the deviations from integers, are decidedly greater than in the examples recorded by Fabry and Perot.

The above results for cadmium suffice to indicate what deviations from integral values are to be expected when any radiation is compared with cadmium red assumed integral. In so far as the expected fractional parts appear in the results, so far are the ratios of wave-lengths assumed in the calculation verified. The following are the wave-lengths, reckoned in air at $15^{\circ} \mathrm{C}$. and $760 \mathrm{~mm}$. pressure, whose ratios to 
cadmium red have been verified by my observations to about one part in a million :-

Cadmium $\left\{\begin{array}{c}6438 \cdot 4722 \\ 5085 \cdot 8240 \\ 4799 \cdot 911\end{array}\right\}$ Michelson.
Mercury $\left\{\begin{array}{l}5790 \cdot 659 \\ 5769 \cdot 598 \\ 5460 \cdot 742 \\ 4358 \cdot 343\end{array}\right\}$ Fabry \& Perot.
Zinc $\left\{\begin{array}{l}6362 \cdot 345 \\ 4810 \cdot 535 \\ 4722 \cdot 164 \\ 4680 \cdot 138 \\ 5895 \cdot 932 \\ 5889.965\end{array}\right\}$ Fabry \& Perot.

I have spoken of an agreement to about 1 part in a million. In several cases the confirmation was decidedly closer. In one only, that of zinc red, did there appear an indication of a disagreement rather outside the limits of error. My observations would point to a wave-length about 1 millionth part greater than that of Fabry and Perot; but in view of the difficulty of observations with the trembler, I am not disposed to insist upon it. The soda observations were on light from a cadmium vacuum-tube in which soda accidentally presented itself. The numbers quoted from Fabry and Perot relate to a soda-flame.

As an example in which the ratios of wave-lengths were less accurately known beforehand, I will give some details relative to helium, beginning with observations of Feb. 9 by the $1 \mathrm{~mm}$. gauge, already referred to. The Table $\mathrm{I}$. annexed gives, in the second column the wave-lengths of the various helium lines recorded by Runge*, in the fifth the same reduced to Michelson's scale as employed by Fabry and Perot. The third column gives the corrections for obliquity as calculated from the observations with the $1 \mathrm{~mm}$. apparatus already recorded, the fourth the differences from the corresponding quantity for cadmium red. Taking, for example, the helium ray of longest wave-length in comparison with cadmium red, we get by (5)

$$
\mathrm{P}^{\prime}=3328 \frac{6438 \cdot 472}{7065 \cdot 22}(1+\cdot 000084)=3033 \cdot 03 \text {. }
$$

These numbers should be integers, were the wave-lengths

* A strophysical Journal, January 1896. 


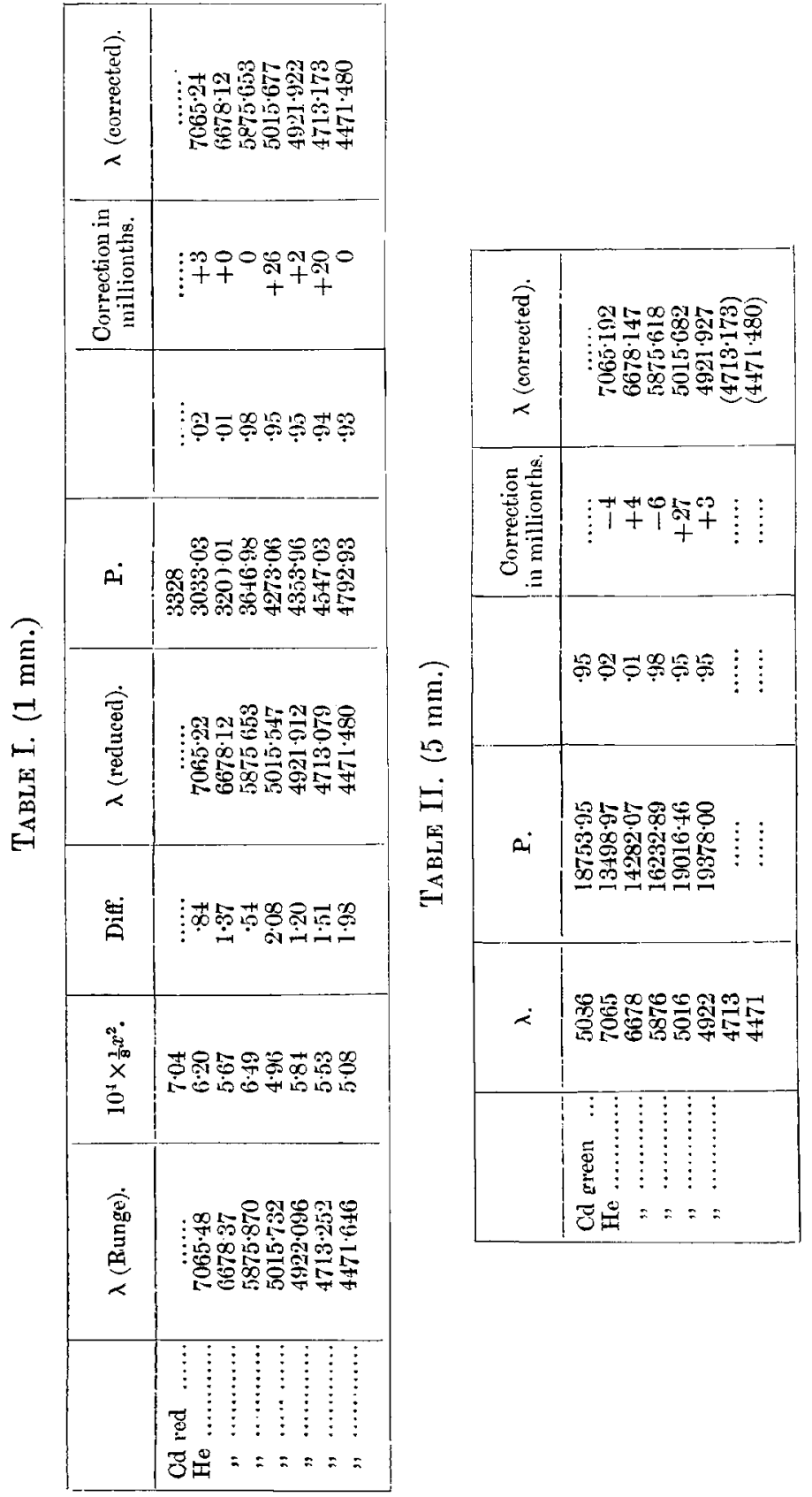


Vibrations of Conducting Surfaces of Revolution.

accurate, and were there no phase change. On account of the phase change as determined from the cadmium observations, the fractional parts should be those entered in the 7th column. The differences are trifling, except in the cases of 5016 and 4713 . The proportional corrections by which the $\lambda$ 's of column 5 are to be increased are set out in column 8 expressed in millionths; but of course an accuracy of 4 or 5 millionths is hardly to be expected in results from a single set of observations with the $1 \mathrm{~mm}$. gauge.

In the observations (Table II.) with the $5 \mathrm{~mm}$. gauge the comparisons were with the cadmium green, for which $\mathrm{P}$ is assumed to be $18753.9 \%$, corresponding to 14814.00 for cadmium red. The numbers given embody the results of three days' observations, but they do not include the wavelengths 4713,4472 . The procedure is the same as for Table I. If the observations with the $5 \mathrm{~mm}$. gauge stood alone, we should be in doubt whether $\mathrm{P}$ for 5016 should be 19016.95 , or 19015.95 . The results with the $1 \mathrm{~mm}$. gauge show that the latter alternative must be chosen. Except in this respect, the $5 \mathrm{~mm}$. results are independent; and they are of course to be preferred as presumably more accurate. The final numbers for helium are therefore those given in column 6 of Table II.

The only further remark that I will make is that the observations on the helium yollow (5876) are not improbably somewhat embarrassed by a companion of feeble luminosity which could not be separated. In the $5 \mathrm{~mm}$. apparatus the two components would be nearly but not quite in coincidence.

LXIII. The Symmetrical Vibrations of Conducting Surfaces of Revolution. By J. W. Nicholson, B.A., M.Sc., Scholar of Trinity College, and Isaac Newton Student in the University of Cambridge*.

T $\mathrm{N}$ a previous paper $\dagger$, the author investigated the possible 1 electrical periods of the space between two perfectly conducting elliptic cylinders belonging to a confocal system. The vibrations of symmetrical type were alone considered. The object of the present paper is to obtain a list of the simpler surfaces of revolution, including cylinders as a particular case, whose vibrations admit readily of analytical treatment, and to examine certain important cases in detail.

* Communicated by the Author.

+ Phil. Mag. Aug. 1905. 